\title{
Nuclear Magnetic Resonance Imaging of Li-ion Battery
}

\author{
D. Ohno, Y. Iwai and J. Kawamura \\ Institute of Multidisciplinary Research for Advanced Materials. Tohoku University, Katahira 2-1-1, Aobaku, \\ Sendai, 980-8577, Japan
}

\section{ARTICLE INFO}

\section{Article history:}

Received 7 October 2010

Received in Revised form 1 December 2010

Accepted 17 December 2010

Keywords:

$\mathrm{LiMn}_{2} \mathrm{O}_{4}$

Lithium compounds

Lithium ion battery

Magnetic resonance imaging

Nuclear magnetic resonance

\begin{abstract}
A B S T R A C T
Nuclear magnetic resonance (NMR) imaging has high sensitivity to proton $\left({ }^{1} \mathrm{H}\right)$ and lithium $\left({ }^{7} \mathrm{Li}\right)$. It is a useful measurement for electrolyte in Li-ion battery. ${ }^{1} \mathrm{H}$ NMR images of lithium ion battery which composed of $\mathrm{LiMn}_{2} \mathrm{O}_{4} / \mathrm{LiClO}_{4}+$ propylene carbonate (PC) / Li-metal have been studied. ${ }^{1} \mathrm{H}$ NMR images of electrolyte near cathode material $\left(\mathrm{LiMn}_{2} \mathrm{O}_{4}\right)$ showed anomalous intensity distribution, which was quite inhomogeneous. From NMR images as a function of repetition time (TR), it was concluded that the anomalous intensity distribution was not due to change of relaxation time but an indirect (spatial) para-magnetization effect from cathode material. The para-magnetization induced by high magnetic field distorts linearity of magnetic gradient field, it produced apparent intensity variance. This functional image is an easy diagnostic measurement for magnetization of cathode material, which has possibility to check a uniformity of cathode material and change of magnetization under electrochemical process.
\end{abstract}

(C) 2010 Atom Indonesia. All rights reserved

\section{INTRODUCTION}

Li-ion battery is an important power source for modern electrical devices such as mobile phone, electric vehicle etc. It is widely researched now. $\mathrm{Li}_{\mathrm{x}} \mathrm{Mn}_{2} \mathrm{O}_{4}$ is typical cathode material which has been receiving increasing attention from several researches as one of the most promising cathode material for Li-ion battery. The reason for this interest resides in its low cost and low toxicity [1]. One of the problem is inhomogeneous cathode material. When $\mathrm{x}=1$, it is an insulator but $\mathrm{x}<1$, it becomes electric conductor. The electrical charge and discharge reaction occur around the part of the conductor. As a result, inhomogeneous charge discharge reaction is happened. Therefore, it is important to understand the change of the local structure.

NMR imaging method is used as a visualization method inside of the Li-ion battery. It has high sensitivity of proton $\left({ }^{1} \mathrm{H}\right)$ and lithium $\left({ }^{7} \mathrm{Li}\right)$. These are included voluminously in the electrolyte. In addition, it is a merit to obtain information on the ion dynamics. On the other hand, the ather methods of visualizing the inside of a battery are shown by $\mathrm{X}$-ray and neutrons tomography. There are many differences between MRI and these methods. One of the differences is that MRI is strongly affected by

\footnotetext{
* Corresponding author.

E-mail address: ohno@mail.tagen.tohoku.ac.jp ( D. Ohno )
}

the magnetic properties. The magnetization changes of $\mathrm{LiMn}_{2} \mathrm{O}_{4}$ is reported by the electrical charge and discharge reaction is reported [2].

In this study, NMR images as a function of several acquisition parameters is reported. The aim of this study is to understand the effect of cathode magnetization caused by MRI effect.

\section{EXPERIMENTAL METHODS}

A cell of Li-ion battery contains nonmagnetic materials as much as possible; ex. a glass tube $(\phi 18.4 \mathrm{~mm})$ as the container, PTFE as the separator and fixing (Fig. 1).

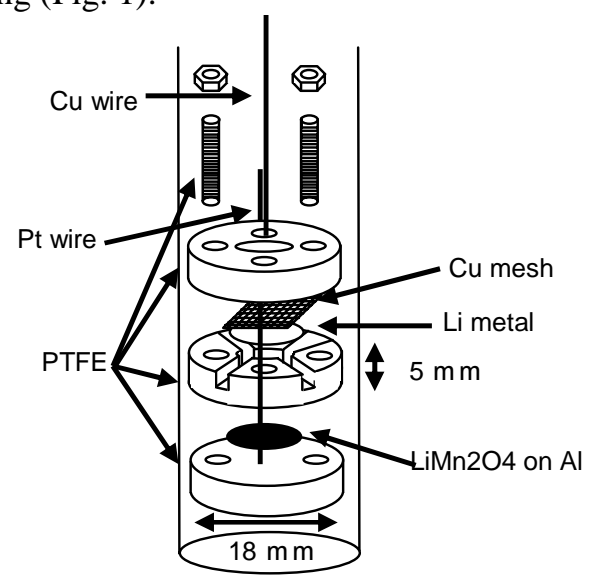

Fig. 1. Schematic representation of model Lithium ion battery. 
It is the purpose of transmission of radiofrequency wave and magnetic gradients applied by MRI coils.

The anode material was lithium metal purchased from Hosen Co., the electrolyte was 1 mole/ $\mathrm{L} \mathrm{LiClO}_{4}$ in propylene carbonate (PC) purchased from Aldrich Co. The cathode was made from $\mathrm{LiMn}_{2} \mathrm{O}_{4}$ powder mixed with polyvinylidene difluoride (PVDF) and the acetylene black at a rate of 85:9:6 (wt \%). After powder and polyvinylpyrrolidone (PVP) were mixed, it was coated on aluminum. A diameter of the aluminium was $12 \mathrm{~mm}$, a thickness of the aluminum and cathode material was $50 \mu \mathrm{m}$ and around $200 \mu \mathrm{m}$, respectively.

Bruker Avance 400 (9.4 T) was used for MRI measurement. The model cell was located in the NMR imaging probe. The resonance frequency of ${ }^{1} \mathrm{H}$ is $400 \mathrm{MHz}$. A pulse sequence for NMR imaging was base on spin-echo method (msme), which is installed in measurement software (paravision) produced by Bruker Biospin Co. Picture size was $25 \mathrm{~mm} \times 25 \mathrm{~mm}$, spatial resolution was $195 \mu \mathrm{m}$ $(128 \times 128$ pixels $)$. The slice thickness was $0.8 \mathrm{~mm}$, number of slice was 7. Echo time (TE) was from $3.495 \mathrm{~ms}$ to $30 \mathrm{~ms}$. Repetition time (TR) was from $300 \mathrm{~ms}$ to $20 \mathrm{~s}$.

\section{RESULTS AND DISCUSSION}

Figure 2 shows $\mathrm{x}-\mathrm{z}$ cross section of Li-ion battery. White parts correspond to proton intensity in liquid electrolyte. The boxes enclosed pink lines correspond to slice position in Fig. 3. $\mathrm{LiMn}_{2} \mathrm{O}_{4}$ is at the bottom of slice, lithium metal is in top of slice [see Fig. 3 (g) and Fig. 3 (a)].

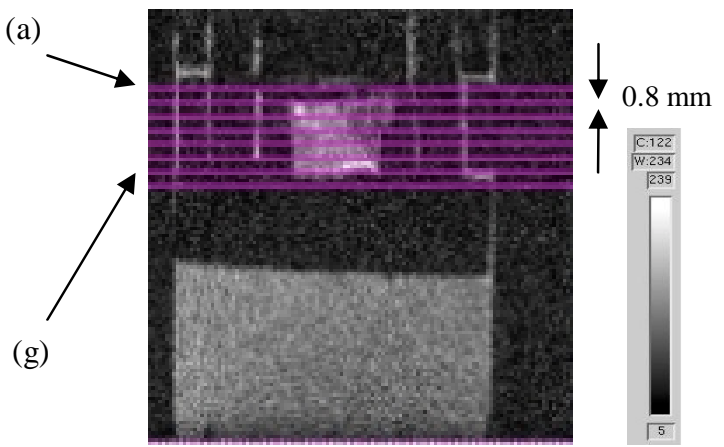

Fig. 2. The $\mathrm{x}-\mathrm{z}$ slice of Li-ion battery taken by spin echo pulse sequence, $\mathrm{FOV}=25 \times 25 \mathrm{~mm}$, matrix size $=128 \times 128$, $\mathrm{TE}=3.495 \mathrm{~ms}, \mathrm{TR}=500 \mathrm{~ms}$, Slice Thickness $=0.8 \mathrm{~mm}$, NS $=4$. The boxes enclosed pink lines correspond to slice position and thickness of $x-y$ slices in Fig. 3.

In Figure 2, the signal intensity between the cathode and anode is not uniform; especially intensity enhancement appeared in the vicinity of $\mathrm{LiMn}_{2} \mathrm{O}_{4}$. To see this effect in detail, $\mathrm{x}$-y slices with different positions are shown in Fig. 3.

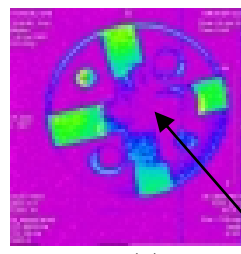

(a)
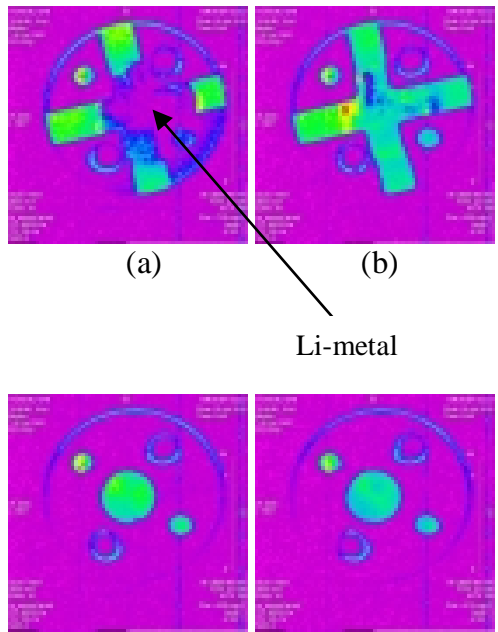

(d)

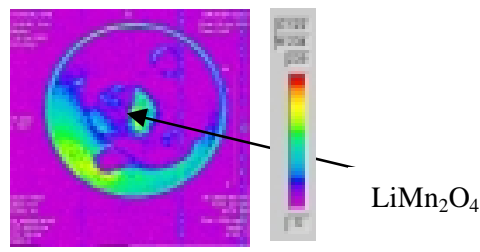

(g) (b)

Li-metal

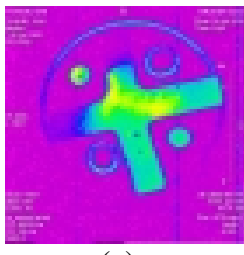

(c)

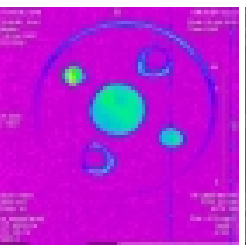

(e)

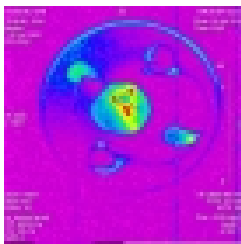

(f)
Fig. 3. The $x-y$ image of Li-ion battery taken by spin echo pulse sequence, $\mathrm{FOV}=25 \times 25 \mathrm{~mm}$, matrix size $=128 \times 128$, $\mathrm{TE}=3.495 \mathrm{~ms}, \mathrm{TR}=500 \mathrm{~ms}$, Slice thickness $=0.8 \mathrm{~mm}$, $\mathrm{NS}=4$. Slice distance $=0.8 \mathrm{~mm}$. The two arrows indicate lithium metal and $\mathrm{LiMn}_{2} \mathrm{O}_{4}$ position.

Figure 3 shows $x-y$ slices of the lithium ion battery, Fig. 3 (a) and (g) correspond to the positions of the lithium metal and $\mathrm{LiMn}_{2} \mathrm{O}_{4}$, respectively. These materials have no intensity because there is no proton $\left({ }^{1} \mathrm{H}\right)$. An interesting phenomenon appeared in the image (f), with a quite inhomogeneous intensity distribution. There are three spots which shows red color in the center, which is a high intensity position, other parts is low intensity.

There are two possibilities for such an intensity enhancement; reduction of relaxation time or distortion of magnetic gradient linearity.

The reduction of relaxation time is caused by elution of paramagnetic materials. These materials have lone pair electrons, which strongly affects the nuclear dipole. This interaction reduces the longitudinal relaxation time $\left(\mathrm{T}_{1}\right)$, leading to a speedy recovery of the equilibrium condition under NMR pulse sequence. It contributes to effective signal detection to be high signal intensity.

Another possibility is distortion of magnetic gradient linearity. A NMR imaging uses linear 
magnetic gradient applied by gradient coil, which play a role of spatial distinction. If the magnetic gradient is truly linear, spatial position and magnetic gradient is one-to-one correspondence however, distortions of linearity from magnetic material eliminate the correspondence, leading to shrinking or expanding of spatial scaling. This effect possibly to produces an apparent intensity enhancement

To understand which effect is dominant, variable TR measurements should provide information for the judgment. TR is a repetition time which is an intermission between pulse sequences. If the TR parameter is long compared with relaxation time $T_{1}$, all nuclear magnetization recover to be equilibrium condition by next acquisition. It means $T_{1}$ reduction effect vanishes if you select long TR value, NMR image would show uniform intensity [3].

Figure 4 shows NMR images taken by various TR values. These intensities are relative intensity. From Fig.4 (b) to (e), the difference is hardly visible in the relative contrast of these images. It indicates that $T_{1}$ reduction effect is not dominant for the intensity enhancement. The intensity of Fig. 4 (a) shows low intensity it is because that too short TR value conducts saturation pulses sequence, which reduces NMR signals.

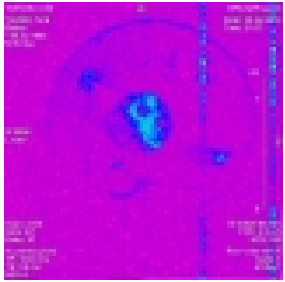

(a) $\mathrm{TR}=100 \mathrm{~ms}$

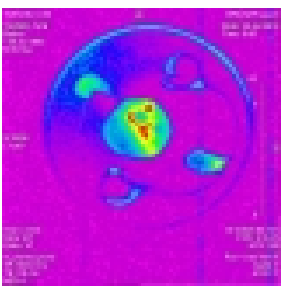

(c) $\mathrm{TR}=500 \mathrm{~ms}$

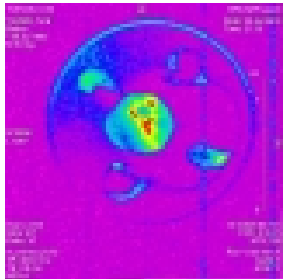

(b) $\mathrm{TR}=300 \mathrm{~ms}$

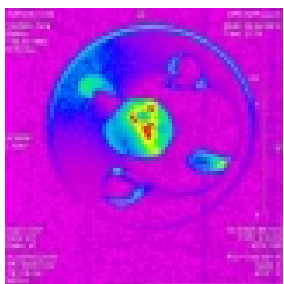

(d) $\mathrm{TR}=1000 \mathrm{~ms}$

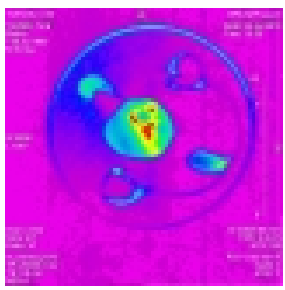

(e) $\mathrm{TR}=20000 \mathrm{~ms}$

Fig. 4. MRI as a function of TR. The $x-y$ image of Li-ion battery taken by spin echo pulse sequence, FOV $=25 \times 25 \mathrm{~mm}$, matrix size $=128 \times 128, \mathrm{TE}=3.495 \mathrm{~ms}$, (a) $\mathrm{TR}=100 \mathrm{~ms}$, (b) $\mathrm{TR}=300 \mathrm{~ms}$, (c) $\mathrm{TR}=500 \mathrm{~ms},(\mathrm{~d}) \mathrm{TR}=1000 \mathrm{~ms}$, (e) $\mathrm{TR}=20.000 \mathrm{~ms}$, Slice thickness $=0.8 \mathrm{~mm}, \mathrm{NS}=4$.

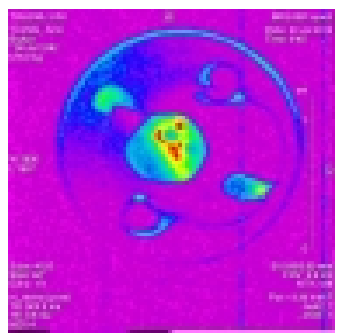

(a) $\mathrm{TE}=3.495 \mathrm{~ms}$

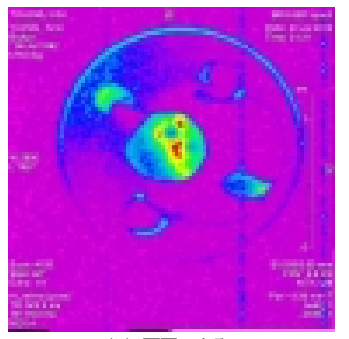

(c) $\mathrm{TE}=15 \mathrm{~ms}$

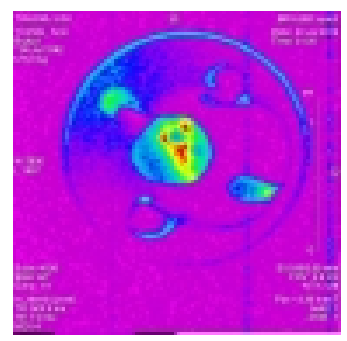

(b) $\mathrm{TE}=7 \mathrm{~ms}$

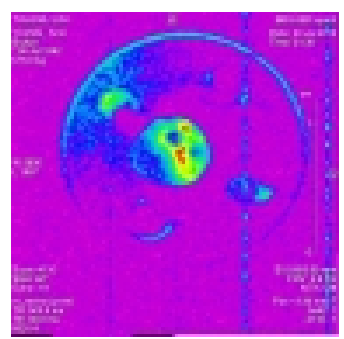

(d) $\mathrm{TE}=30 \mathrm{~ms}$
Fig. 5. MRI as a function of TE. The $x-y$ image of Li-ion battery taken by spin echo pulse sequence, FOV $=25 \times 25 \mathrm{~mm}$, matrix size $=128 \times 128, \mathrm{TR}=500 \mathrm{~ms}$, (a) $\mathrm{TE}=3.495 \mathrm{~ms}$, (b) $\mathrm{TR}=7 \mathrm{~ms}$, (c) $\mathrm{TR}=15 \mathrm{~ms}$, (d) $\mathrm{TR}=30 \mathrm{~ms}$, Slice thickness $=0.8 \mathrm{~mm}, \mathrm{NS}=4$.

Figure 5 shows NMR images taken by various TE values. TE is an echo time of $90-180$ degree pulse sequence. An analogy of variable TR measurement, it detects difference of $T_{2}$ in each part [3]. In Fig. 5, the difference is hardly visible in the contrast of these images. These also agree with our conclusion that the intensity enhancement is not caused by changing the relaxation time. From NMR images with various TR and TE values, it is concluded that intensity enhancement is caused by the magnetization of $\mathrm{LiMn}_{2} \mathrm{O}_{4}$. This cathode material is known as paramagnetic material [2]. Magnetizations induced by external high magnetic field $(9.4 \mathrm{~T})$ add extra magnetic field to linear gradient field $[3,4]$, leading to distortion of spatial correspondences. This effect causes intensity enhancement. However, this is only a qualitative description. More concrete calculation between distortion of gradient field linearity and intensity enhancement will be published elsewhere.

Another characteristic phenomenon is the intensity distribution, there are three spots which have high intensity but other parts have intensity. This is probably due to inhomogeneous a reduced cathode material, so that mixture of $\mathrm{LiMn}_{2} \mathrm{O}_{4}$, PVDF and acetylene black is not uniform. A part which contain a large quantity of $\mathrm{LiMn}_{2} \mathrm{O}_{4}$ has high magnetism, however, low concentration parts are having less magnetism. Only high magnetic parts disturb linearity of gradient field strongly, which leads to intensity enhancement. 
This study presents that NMR signal is quite sensitive to magnetism. It shows different intensity from without magnetic material case. This is an artifact, which should be removed in a normal situation however, when you consider the alternative, looking at the positive side of this artifact, it is a functional image. From such point of view, NMR imaging technique has several capabilities of detecting magnetism in materials by means of ${ }^{1} \mathrm{H}$ NMR images. This method has spatial information in its nature; therefore it is quite easy to detect distribution of magnetism in a matter. Moreover, it can detect spatial information not only near but also apart from material. It means NMR images of magnetic material can detect distance dependence of magnetism.

Hopefully, this functional NMR image will contribute the mechanisms of lithium ion battery.

\section{CONCLUSIONS}

${ }^{1} \mathrm{H}$ NMR images of lithium ion battery which composed $\mathrm{LiMn}_{2} \mathrm{O}_{4} / \mathrm{LiClO}_{4}+$ propylene carbonate (PC) / Li-metal have been studied. ${ }^{1} \mathrm{H}$ NMR images of electrolyte near cathode material $\left(\mathrm{LiMn}_{2} \mathrm{O}_{4}\right)$ showed anomalous intensity enhancement. Relaxation Time dependent NMR images show that this anomaly is not caused by the reduction of the relaxation time but is an indirect (spatial) magnetization effect from the cathode material. The para-magnetization induced by high magnetic field distorts the linearity of magnetic gradient field, it produced apparent intensity variance.
Another characteristic phenomenon is intensity distribution. It was probably due to inhomogenity in the cathode material. A part which contained lots of $\mathrm{LiMn}_{2} \mathrm{O}_{4}$ had high magnetism however, low concentration parts were less magnetism. Only high magnetic parts disturb linearity of gradient field strongly, which lead to intensity enhancement.

There is a possibility that the change in the magnetization of a cathode by the electrical charge and discharge reaction can be confirmed.

\section{ACKNOWLEDGMENT}

This work belongs to Research and Development Initiative for Scientific Innovation of New Generation Batteries (RISING) project with New Energy and Industrial Technology Development Organization (NEDO).

\section{REFERENCES}

1. G. Li, Y. Iijima, T. Kudo and H. Azuma, Solid State Ionics 146 (2002) 5.

2. Y.I. Jang, B. Huang, F.C. Chou, D.R. Sadoway and Y.M. Chiang, J. Appl. Phys. 87 (2000) 7382 .

3. K. Kose, NMR Imaging, $1^{\text {st }}$ ed., Kyoritsu Publ., Japan (2004) 70.

4. E.M. Haacke, R.W. Brown, M.R. Thompson, R. Venkatesan, Magnetic Resonance Imaging: Physical Principles and Sequence Design, $1^{\text {st }}$ ed., Wiley-Liss, US (1999) 614. 